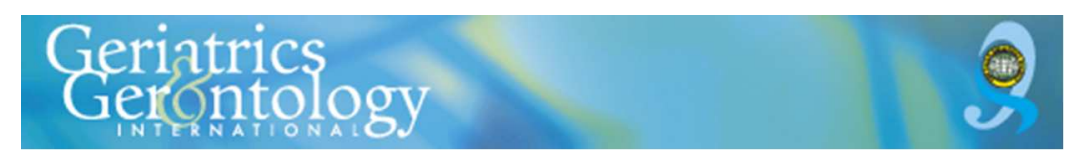

\title{
Social participation and the onset of hypertension among middle-aged and old population: Longitudinal evidence from the China Health and Retirement Longitudinal Study
}

\begin{tabular}{|c|c|}
\hline Journal: & Geriatrics \& Gerontology International \\
\hline Manuscript ID & GGI-0602-2017 \\
\hline Manuscript Type: & Original Article \\
\hline Date Submitted by the Author: & 05-Sep-2017 \\
\hline Complete List of Authors: & $\begin{array}{l}\text { Tu, Raoping; Nagasaki University, Department of International Health, } \\
\text { Institute of Tropical Medicine (NEKKEN); Nagasaki Unniversty, Leading } \\
\text { Program, Graduate School of Biomedical Sciences } \\
\text { Inoue, Yosuke; University of North Carolina at Chapel Hill, Carolina } \\
\text { Population Center } \\
\text { Yazawa, Aki; University of Fukui, Research Center for Child Mental } \\
\text { Development } \\
\text { Hao, Xiaoning; China National Health and Development Research Center } \\
\text { Li, Yueping; Fujian Medical University, Department of Health Management, } \\
\text { School of Public Health } \\
\text { Lin, Xinquan; Fujian Provincial Center for Disease Control and Prevention, } \\
\text { Department for Chronic and Non-communicable Disease Control and } \\
\text { Prevention } \\
\text { He, Fei; Fujian Medical University, Department of Epidemiology, School of } \\
\text { Public Health } \\
\text { Yamamoto, Taro; Nagasaki University, Department of International Health, } \\
\text { Institute of Tropical Medicine (NEKKEN) }\end{array}$ \\
\hline Keywords: & $\begin{array}{l}\text { Hypertension < Geriatric Medicine }<\text { Clinical Medicine, Public Health }< \\
\text { Basic Science, Epidemiology }<\text { Socio-Medical Science }\end{array}$ \\
\hline Optional Keywords: & Blood pressure, Longitudinal studies, Social participation \\
\hline
\end{tabular}

\section{SCHOLARONE ${ }^{\text {m }}$}

Manuscripts 
Title:

Social participation and the onset of hypertension among middle-aged and old population: Longitudinal evidence from the China Health and Retirement Longitudinal Study

Authors:

Raoping $\mathrm{Tu}^{1,2}$, Yosuke Inoue ${ }^{3 *}$, Aki Yazawa ${ }^{4}$, Xiaoning $\mathrm{Hao}^{5}$, Yueping $\mathrm{Li}^{6}$, Xiuquan $\mathrm{Lin}^{7}$, Fei $\mathrm{He}^{8}$, Taro Yamamoto $^{1}$

${ }^{1}$ Department of International Health, Institute of Tropical Medicine (NEKKEN), Nagasaki University, Nagasaki 852-8523, Japan

${ }^{2}$ Leading Program, Graduate School of Biomedical Sciences, Nagasaki University, Nagasaki 852-8523, Japan

${ }^{3}$ Carolina Population Center, The University of North Carolina at Chapel Hill, Chapel Hill, NC 27516, USA

${ }^{4}$ Research Center for Child Mental Development, University of Fukui, 23-3 Matsuoka-Shimoaizuki, Eiheijicho, Yoshida-gun, Fukui 910-1193, Japan

${ }^{5}$ China National Health and Development Research Center, Beijing 100191, China

${ }^{6}$ Department of Health Management, School of Public Health, Fujian Medical University, Fujian 350122, China

${ }^{7}$ Department for Chronic and Non-communicable Disease Control and Prevention, Fujian Provincial Center for Disease Control and Prevention, Fujian 350001, China

${ }^{8}$ Department of Epidemiology, School of Public Health, Fujian Medical University, Fujian 350122, China

* Correspondence to: Yosuke Inoue

Carolina Population Center, The University of North Carolina at Chapel Hill, Chapel Hill, NC 27516, USA;

E-mail: yosuke.yoshi.yosky@gmail.com

Phone: 1-919-593-3765

Short running title: Social participation and hypertension 
Keywords: Blood Pressure; Longitudinal Studies; Social Participation 
Text:

\section{Introduction}

Hypertension is one of the most important risk factors of cardiovascular diseases. ${ }^{1}$ Globally, it has been estimated that there were 1.39 billion people living with hypertension in $2010^{2}$ and this figure was projected to increase to 1.56 billion in $2025 .{ }^{1}$ It is of note that the prevalence of hypertension has been increasing disproportionately in developing countries ${ }^{2}$, such as China. For example, the prevalence of hypertension increased from $11.3 \%$ to $33.6 \%$ during the period between 1991 and 2010 in China $^{3}$, which was possibly caused by rapid urbanization, westernization of lifestyle and population aging of the country. ${ }^{4}$

While previous research in China has focused on the association between hypertension and individual lifestyle-related risk factors, such as obesity ${ }^{5}$, alcohol consumption ${ }^{6}$, smoking $^{7}$, physical activity ${ }^{8}$ and $\operatorname{diet}^{9}$, comparatively little attention has been paid to the role of social participation (i.e., participation in community organizations). This refers to involvement in the activities of formal and informal societal groups ${ }^{10}$ and has recently attracted broad attention from public health researchers and practitioners in relation to promoting healthy aging and creating age-friendly cities. ${ }^{11}$

Several research has demonstrated a protective role of social participation on hypertension in developed countries. For example, Yazawa et al. ${ }^{12}$ showed that participation in horizontal organizations (i.e., groups that are characterized by non-hierarchical, egalitarian relationships among group members; volunteer, sport and hobby groups in their study) was inversely associated with hypertension among older people in Japan. Kamiya et al. ${ }^{13}$ showed that participating in a greater number of organizations was associated with a lower odds of hypertension among older population living in the U.K.

This study was designed to extend the previous studies in several ways. First, we used a longitudinal dataset and investigated the association to deal with the issue of reverse causality. Previous studies used crosssectional design to investigate the association. ${ }^{12,13}$ Second, few study was conducted in China, which exemplifies rapid economic development and population aging in the last several decades better than any other country and thus, the increasing trend of hypertension. ${ }^{4,}{ }^{14}$ Social participation could be a potential cost- 
effective means to help mitigate disease burden associated with hypertension in China which must meet the demand of a rapidly aging population with limited financial and medical resources.

The aim of this study, therefore, is to investigate the longitudinal association between social participation and hypertension in China, using data from the China Health and Retirement Longitudinal Study (CHARLS), which is a nationally representative survey of the middle and old-aged people in China.

\section{Methods \\ Data}

CHARLS is a nationally representative longitudinal study which was conducted in Waves 2011 and $2013 .{ }^{15}$ As shown in Figure 1, out of 17,638 people who participated in Wave 2011, we excluded those with missing information on blood pressure $(n=3,925)$ and covariates $(n=69)$. We further excluded those who were hypertensive in Wave $2011(\mathrm{n}=5,584)$. Out of 8,060 participants who were not hypertensive in Wave 2011, 7,434 people participated in Wave 2013. After excluding those who did not provide information on blood pressure in Wave $2013(\mathrm{n}=1,951)$, we had an analytic sample of 5,483 people $(2,591$ men and 2,892 women).

The current study was a secondary analysis of the CHARLS dataset, which is publicly available (http://charls.pku.edu.cn/en). The study protocol of the CHARLS was originally approved by the Ethical Review Committee of Peking University, China. Subjects gave informed consent for participation.

\section{Dependent variable}

Blood pressure was measured at participants' houses by trained interviewers. It was measured three times on their left arm with 45-second intervals. Participants were requested to sit down with their left arm placed on the table and palm facing up. Participants were considered to be hypertensive if systolic blood pressure (SBP) $\geq$ $140 \mathrm{mmHg}$, diastolic blood pressure (DBP) $\geq 90 \mathrm{mmHg}$, or having self- reported taking antihypertensive medication. ${ }^{16}$

\section{Independent variables}


Social participation at baseline was defined by participants saying yes to participating in any of the following activities once a week or more frequently in the previous one month: (1) interact with friends; (2) play mahjong (i.e., a Chinese tile-based game), chess or cards, or go to a community club; (3) go to a sport, social, or other kind of club; (4) take part in a community-related organization; and (5) attend volunteer or charity work.

\section{Covariates}

Baseline information collected on respondents' socio-demographic characteristics include: age (in years), marital status (married; unmarried), educational attainment ( $<9$ years; 9 years; $>9$ years). Body mass index (BMI) was calculated as weight (in $\mathrm{kg}$ ) divided by squared height $\left(\mathrm{m}^{2}\right)$; it was then categorized in the following categories: $<18.5$ (underweight); 18.5 - 23.99 (normal); $24-27.99$ (overweight); and $\geq 28$ (obese). ${ }^{17}$ Location of residence (urban / rural) was also incorporated into models to account for urban/rural differences in the association between social participation and health. ${ }^{18}$

The health-related behaviors include smoking status (never smoke; used to smoke; and currently smoke), alcohol consumption (never drink; less than once a month; and once a month or more frequently), selfreported poor health (1: very poor or poor; 0 : fair, good or very good) and depressive symptoms (evaluated with the 10-item version of the Center for Epidemiologic Studies Depression Scale (CES-D 10). The CES-D 10 score ranges from 0 to 30 and participants were categorized into two groups based on their scores (i.e., CES-D $10<10$ : no depressive symptoms; CES-D $10 \geq 10$ : depressive symptoms). ${ }^{19}$

\section{Statistical analysis}

Descriptive characteristics of the study participants were compared by presence of hypertension in 2013 using $\chi^{2}$ tests for each sex. We used a multilevel Poisson regression model with a robust variance estimator to investigate the association between social participation and hypertension while accounting for multiple individuals within community. We stratified the analysis by sex since previous research suggested that the health impact of social participation may differ by sex. ${ }^{20}$ We also incorporated sampling weights to produce nationally representative estimates of variables. Those who did not provide information on depressive 
symptoms and BMI were categorized as "missing" in order to retain statistical power.

We used Stata 13.0 (Stata Corp, College Station, TX, USA) for statistical analyses. Results are presented as incidence rate ratios (IRRs) with 95\% confidence intervals (CI). The level of statistical significance was set at $\mathrm{p}<0.05$ (two-tailed).

\section{Results}

Table 1 shows the baseline characteristics of the study participants stratified by sex and presence of hypertension in Wave 2013. Out of 5,483 participants, $52.7 \%$ were women, and $20.6 \%$ and $17.2 \%$ of men and women respectively developed hypertension during the period between 2011 and 2013. Those who became hypertensive in 2013 tended to be older $(\mathrm{p}<0.001)$, not married among women $(\mathrm{p}<0.001)$, less educated among men $(p=0.053)$, more obese $(p=0.006$ for men and $p=0.001$ for women $)$ and less likely to participate in community organizations among women $(\mathrm{p}=0.070)$. There was no marked difference in relation to location or residence (urban or rural), smoking status, alcohol consumption, self-reported poor health and depressive symptoms.

Table 2 shows results of a Poisson regression model investigating the association between social participation and the onset of hypertension among men and women. Among men, social participation was not statistically significantly associated with the onset of hypertension. Age was positively associated with the onset of hypertension; for example, those aged 70 years or older had 1.82 times higher the IRR of hypertension compared to those aged 45 to 49 (reference category). Those who were more educated were less likely to become hypertensive (e.g., schooling of more than 9 years: $\mathrm{IRR}=0.74,95 \% \mathrm{CI}=0.55-0.98$ ). A higher frequency of alcohol consumption was positively associated with the onset of hypertension (IRR $=1.20$, $95 \% \mathrm{CI}=1.01-1.42$ ). Among women, social participation was inversely associated with the onset of hypertension $(\mathrm{IRR}=0.80,95 \% \mathrm{CI}=0.67-0.95)$. In addition, higher age (e.g., those aged 70 years or older: $\mathrm{IRR}=3.00,95 \% \mathrm{CI}=2.22-4.05)$ and higher $\mathrm{BMI}(\geq 28: \mathrm{IRR}=2.30,95 \% \mathrm{CI}=1.60-3.30)$ were associated with the onset of hypertension. 


\section{Discussion}

Using nationally representative data from among the middle and old-aged Chinese population, this prospective study revealed that among women, participating in community organizations once a week or more frequently was inversely associated with the onset of hypertension. On the other hand, no such association was observed among men.

The inverse association between social participation and hypertension observed in this study was in line with Yazawa et al. ${ }^{12}$ who showed in their cross-sectional study in Japan that participating in horizontal organizations (i.e., groups that are characterized by non-hierarchical, egalitarian relationships among group members; volunteer, sport and hobby groups in their study) once a month or more frequently was associated with a lower prevalence of hypertension among the older Japanese population ( $\geq 65$ years old). As we used information on similar types of community organizations/activities (e.g., interaction with friends, community club, a sport, social, or other kind of club, community-related organization, and voluntary or charity work) to those included in Yazawa et al., this study extends their study by providing longitudinal evidence of the association between social participation and hypertension.

Several possible mechanisms linking social participation and hypertension have been proposed, which can be divided into two potential aspects (i.e., health behavior and psychological stress). For health behavior, Lindström et al. ${ }^{10}$ used data from the Malmö Diet and Cancer Study which involved 11,837 participants in Sweden and showed that social participation (defined by taking part in the activities of 13 formal and informal groups in society) was associated with elevated level of light physical activity. They also showed that health behavior was positively associated with vegetable and fruit consumption ${ }^{21}$ and smoking cessation ${ }^{22}$ among the same study population. In terms of psychological stress, the association between social participation and hypertension may operate through providing a sense of companionship and belonging, which could enhance people's ability to cope with stressors. ${ }^{23,24}$ Future study should focus on elucidating these detailed mechanisms in the Chinese context to better inform the development of intervention techniques.

We did not observe a statistically significant association among men. Sex differences in the association between social participation and health outcomes have been reported in previous literature as well. For 
example, in Japan, participation in social activities was associated with a relatively stable transition of instrumental activities of daily living and cognitive function among women but not among men. ${ }^{25,}{ }^{26}$ Another study undertaken in Korea showed that participation in formal and informal group activities had a protective effect on self-rated health among old people, which was larger among women than men. ${ }^{20}$

While the exact mechanism has yet to be determined, there are several possible reasons to explain the null findings among men. First, we can hypothesize that men are less affected by a neighborhood environment due to comparatively less time spent in the community ${ }^{27}{ }^{28}$; for instance, Stafford et al. ${ }^{27}$ investigated the association between a variety of neighborhood environmental indicators and self-reported health in U.K. and showed that the effects were larger in women than in men. While social participation was operationalized as an individual-level variable in this study, it can be hypothesized that social or physical environments which engender more social participation also affect health, particularly health of individuals who stay longer in the environment (i.e., women). Another possibility is that men are more likely to be in key positions of the community organizations which could be a psychological stressor offsetting the beneficial health effect of social participation. Some previous studies also reported that frequent participation in community organizations does not always have a health protecting effect. ${ }^{29}$ However, it should also be noted that Takagi et al. ${ }^{18}$ reported that occupying a key positions within an organization rather promoted mental health of Japanese old men, interpreting that men might feel being rewarded by seeking authoritative positions in their Japanese culture. Future study should focus on more detailed pathways linking social participation and hypertension.

The findings of our study have important public health implications. Given that China's social and medical infrastructure must meet the demands of a rapidly growing, aging population, the government should implement an efficient and cost-effective policy, which can help mitigate morbidity and mortality associated with hypertension. In this respect, promoting social participation could be one of the possible solutions. Several studies in China investigating the association between social participation and health outcomes other than hypertension and showed a beneficial effect of social participation. For example, Li et al. ${ }^{30}$ showed that participating in volunteer group was associated with lower depressive symptoms, less functional limitation and better self-reported health among urban Chinese.

There are several limitations to this study. First, study participants in this study might not have fully 
represented the Chinese middle and old-aged population. For example, an estimated 260 million people migrant workers in $2010^{4}$ may not have been accurately represented in this population-based survey. Given that they have limited access to social and medical services and are subject to discrimination and stigmatization ${ }^{4}$, this omission might have biased our results. Another possibility is that those who were hypertensive might feel reluctant to participate in the survey. Second, there are several other variables which would have better explained the association between social participation and hypertension, such as information on how long they had participated in community organizations, physical activity level, diet (e.g., sodium and potassium consumption), performance of the key role in the community organizations and geographic location of residence.

In conclusion, our study suggests that participating in community organizations more than once a week or more frequently reduces the onset of hypertension among middle and old-aged populations in China, particularly among women. Promoting social participation might be one of the productive ways to mitigate the disease burden associated with hypertension in China.

\section{Acknowledgments}

This study used data from the China Health and Retirement Longitudinal Study (CHARLS) conducted by the National School of Development at Peking University, China. R.P.T. is supported by the Program for Nurturing Global Leaders in Tropical and Emerging Communicable Diseases at Graduate School of Biomedical Sciences, Nagasaki University. Y.I. is financially supported by the Nippon Foundation International Fellowship Program (http://intl-fellow.jp/).

\section{Disclosure statement}

The authors declare that they have no competing interests.

\section{References}

[1] Kearney PM, Whelton M, Reynolds K, Muntner P, Whelton PK, He J. Global burden of hypertension: 
analysis of worldwide data. Lancet 2005; 365: 217-23.

[2] Mills KT, Bundy JD, Kelly TN, et al. Global Disparities of Hypertension Prevalence and Control: A Systematic Analysis of Population-based Studies From 90 Countries. Circulation 2015; 132.

[3] Bundy JD, He J. Hypertension and Related Cardiovascular Disease Burden in China. Ann Glob Health 2016; 82: 227-33.

[4] Gong P, Liang S, Carlton EJ, et al. Urbanisation and health in China. Lancet 2012; 379: 843-52.

[5] Wang Y, Mi J, Shan X, Wang QJ, Ge K. Is China facing an obesity epidemic and the consequences? The trends in obesity and chronic disease in China. Int J Obesity 2007; 31: 177.

[6] Li Z, Bai Y, Guo X, Zheng L, Sun Y, Roselle AM. Alcohol consumption and cardiovascular diseases in rural China. Int J Cardiol 2016; 215: 257-62.

[7] Wang JW, Zhang LX, Wang F, Liu LS, Wang HY, D CNSCK. Prevalence, Awareness, Treatment, and Control of Hypertension in China: Results From a National Survey. Am J Hypertens 2014; 27: 1355-61.

[8] Hong YL, Bots ML, Pan XW, et al. Physical-Activity and Cardiovascular Risk-Factors in Rural Shanghai, China. Int J Epidemiol 1994; 23: 1154-58.

[9] Wang M, Moran AE, Liu J, et al. Effect of Dietary Salt Restriction on Blood Pressure in Chinese Adults: A Meta-Analysis. Circulation 2014; 130.

[10] Lindström M, Hanson BS, Östergren P-O. Socioeconomic differences in leisure-time physical activity: the role of social participation and social capital in shaping health related behaviour. Soc Sci Med 2001; 52: 441-51.

[11] World Health Organization. Global age-friendly cities: A guide., 2007.

[12] Yazawa A, Inoue Y, Fujiwara T, et al. Association between social participation and hypertension among older people in Japan: the JAGES Study. Hypertens Res 2016.

[13] Kamiya Y, Whelan B, Timonen V, Kenny RA. The differential impact of subjective and objective aspects of social engagement on cardiovascular risk factors. BMC Geriatrics 2010; 10: 81.

[14] Yang GH, Kong LZ, Zhao WH, et al. Emergence of chronic non-communicable diseases in China. Lancet 2008; 372: 1697-705.

[15] Zhao Y, Hu Y, Smith JP, Strauss J, Yang G. Cohort profile: The China health and retirement 
longitudinal study (CHARLS). Int J Epidemiol 2012; 43: 61-68.

[16] Gu DF, Reynolds K, Wu XG, et al. Prevalence, awareness, treatment, and control of hypertension in China. Hypertension 2002; 40: 920-27.

[17] Zhou B. Effect of body mass index on all-cause mortality and incidence of cardiovascular diseases-report for meta-analysis of prospective studies open optimal cut-off points of body mass index in Chinese adults. Biomed Environ Sci 2002; 15: 245-52.

[18] Takagi D, Kondo K, Kawachi I. Social participation and mental health: moderating effects of gender, social role and rurality. BMC Public Health 2013; 13: 701.

[19] Chen H, Mui AC. Factorial validity of the Center for Epidemiologic Studies Depression Scale short form in older population in China. Int Psychogeriatr 2014; 26: 49-57.

[20] Lee HY, Jang S-N, Lee S, Cho S-I, Park E-O. The relationship between social participation and selfrated health by sex and age: a cross-sectional survey. Int J Nurs Stud 2008; 45: 1042-54.

[21] Lindström M, Hanson BS, Wirfält E, Östergren P-O. Socioeconomic differences in the consumption of vegetables, fruit and fruit juices: The influence of psychosocial factors. Eur J Public Health 2001; 11: 5159.

[22] Lindström M, Hanson BS, Östergren P-O, Berglund G. Socioeconomic differences in smoking cessation: the role of social participation. Scand J Soc Med 2000; 28: 200-08.

[23] Yang YC, Boen C, Mullan Harris K. Social relationships and hypertension in late life: evidence from a nationally representative longitudinal study of older adults. $J$ Aging Health 2015; 27: 403-31.

[24] Hyyppä MT, Mäki J. Social participation and health in a community rich in stock of social capital. Health Educ Res 2003; 18: 770-79.

[25] Tomioka K, Kurumatani N, Hosoi H. Association Between Social Participation and 3-Year Change in Instrumental Activities of Daily Living in Community-Dwelling Elderly Adults. J Am Geriatr Soc 2017; 65: 107-13.

[26] Tomioka K, Kurumatani N, Hosoi H. Social participation and cognitive decline among communitydwelling older adults: a community-based longitudinal study. J Gerontol B Psychol Sci Soc Sci 2016: gbw059. [27] Stafford M, Cummins S, Macintyre S, Ellaway A, Marmot M. Gender differences in the associations 
between health and neighbourhood environment. Soc Sci Med 2005; 60: 1681-92.

[28] Inoue Y, Stickley A, Yazawa A, et al. The association between economic development, lifestyle differentiation, and C-reactive protein concentration within rural communities in Hainan Island, China. Am $J$ Hum Biol 2016; 28: 186-96.

[29] Iwase T, Suzuki E, Fujiwara T, Takao S, Doi H, Kawachi I. Do bonding and bridging social capital have differential effects on self-rated health? A community based study in Japan. J Epidemiol Community Health 2012; 66: 557-62.

[30] Li YW, Xu L, Chi I, Guo P. Participation in Productive Activities and Health Outcomes Among Older Adults in Urban China. Gerontologist 2014; 54: 784-96. 
Figure legend

Figure 1. CHARLS participant flow chart (2011-2013)

1

2

4

5

6

10

11

12

13

14

15

16

17

18

19

20

21

22

23

24

25

26

27

28

29

30

31

32

33

34

35

36

37

38

39

40

41

42

43

44

45

46

47

48

49

50

51

52

53

54

55

56

57

58

59

60 
Table 1. Baseline characteristics of the study participants in the China Health and Retirement Longitudinal Study (CHARLS) (2011)

\begin{tabular}{|c|c|c|c|c|c|c|}
\hline & Men ( & ,591) & & Women & $=2,892)$ & \\
\hline & $\begin{array}{l}\text { Not hypertensive } \\
\text { in } 2013(n=2,057)\end{array}$ & $\begin{array}{c}\text { Hypertensive } \\
\text { in } 2013(n=534)\end{array}$ & p-value ${ }^{a}$ & $\begin{array}{l}\text { Not hypertensive } \\
\text { in } 2013(n=2,394)\end{array}$ & $\begin{array}{c}\text { Hypertensive } \\
\text { in } 2013(n=498)\end{array}$ & p-value ${ }^{a}$ \\
\hline Age, n (\%) & & & & & & \\
\hline $45-49$ & 388 (18.9) & $74(13.9)$ & $<0.001$ & $746(31.2)$ & $104(20.9)$ & $<0.001$ \\
\hline $50-59$ & $788(38.3)$ & $179(33.5)$ & & $929(38.8)$ & $170(34.1)$ & \\
\hline $60-69$ & $625(30.4)$ & $178(33.3)$ & & $550(23.0)$ & $137(27.5)$ & \\
\hline $70+$ & $256(12.4)$ & $103(19.3)$ & & $169(7.1)$ & $87(17.5)$ & \\
\hline Marital status (married), n (\%) & $1910(92.9)$ & $485(90.8)$ & 0.114 & $2153(89.9)$ & 418 (83.9) & $<0.001$ \\
\hline Education, n (\%) & & & & & & \\
\hline$<9$ years & $1210(58.8)$ & $342(64.0)$ & 0.053 & $1815(75.8)$ & $391(78.5)$ & 0.171 \\
\hline 9 years & $552(26.8)$ & $133(24.9)$ & & $387(16.2)$ & 79 (15.9) & \\
\hline$>9$ years & $295(14.3)$ & $59(11.0)$ & & $192(8) .0$ & $28(5.6)$ & \\
\hline
\end{tabular}

Location of residence, $\mathrm{n}(\%)$ 


\section{Urban \\ Rural}

Smoking, n (\%)

Never

Quit

Smoke

Alcohol consumption, $\mathrm{n}(\%)$

Don't drink

Less than once a month

Once a month or more frequently

Body mass index, $\mathrm{n}(\%)$

$$
\begin{aligned}
& <18.5 \\
& 18.5-23.99 \\
& 24-27.99 \\
& \geq 28
\end{aligned}
$$

Missing

Self-rated health (poor), n (\%)

\begin{abstract}
$336(16.3)$
\end{abstract}
$1721(83.7)$

$102(19.1)$

$179(33.5)$

$535(26.0)$

$307(14.9)$

$1215(59.1)$

$912(44.3)$

244 (11.9)

$901(43.8)$

157 (7.6)

1375 (66.8)

400 (19.4)

83 (4.0)

$42(2.0)$

481 (23.4)

318 (59.6)

222 (41.6)

$54(10.1)$

$258(48.3)$

$47(8.8)$

$315(59)$

124 (23.2)

28 (5.2)

$20(3.7)$

115 (21.5)
0.129

441 (18.4)

1953 (81.6)
$2222(92.8)$

$36(1.5)$

$136(5.7)$

0.150

0.789

$063(86.2)$

$143(6.0)$

188 (7.9)

181 (7.6)

$1264(52.8)$

$706(29.5)$

$191(8.0)$

$52(2.2)$

705 (29.4)
$96(19.3)$

$402(80.7)$

455 (91.4)

$14(2.8)$

$29(5.8)$

$438(88.0)$

$29(5.8)$

$31(6.2)$

$33(6.6)$

0.001

$230(46.2)$

159 (31.9)

67 (13.5)

$9(1.8)$

$138(27.7)$
0.123

0.447

0.655

447

0.438 
Depressive symptoms, $\mathrm{n}(\%)$

\begin{tabular}{|c|c|c|c|c|c|c|}
\hline Not depressed & $1358(66)$ & $369(69.1)$ & 0.370 & $1278(53.4)$ & $268(53.8)$ & 0.669 \\
\hline Depressed & $601(29.2)$ & $144(27)$ & & 975 (40.7) & $196(39.4)$ & \\
\hline Missing & $98(4.8)$ & $21(3.9)$ & & $141(5.9)$ & $34(6.8)$ & \\
\hline \multicolumn{7}{|l|}{ Social participation, n (\%) } \\
\hline Less than once a week & $1418(68.9)$ & $354(66.3)$ & 0.242 & $1567(65.5)$ & 347 (69.7) & 0.070 \\
\hline
\end{tabular}

${ }^{\text {a }}$ Characteristics of the participants were compared using $\chi^{2}$ test. 
Table 2. Results of sex-stratified multilevel Poisson regression model investigating the association between social participation and the incidence of hypertension in China (2011-2013)

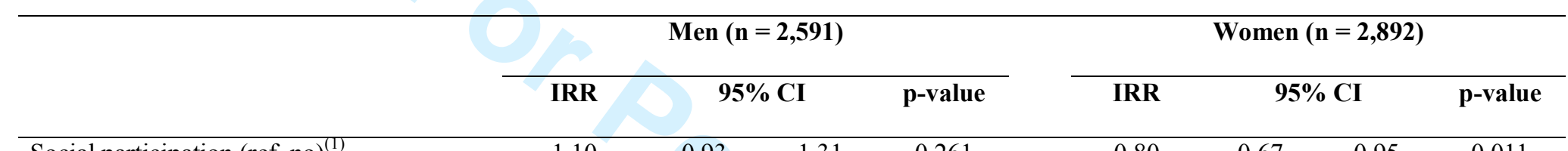

Social participation (ref. no) ${ }^{(1)}$

1.10

$0.93 \quad 1.31 \quad 0.261$

0.80

0.67

0.95

Age group (ref. 45 - 49)

$$
\begin{aligned}
& 50-59 \\
& 60-69 \\
& 70+
\end{aligned}
$$

Marital status (ref. unmarried)

0.91

$1.49 \quad 0.213$

$1.31 \quad 1.04$

1.66

0.022

1.35

1.06

$1.73 \quad 0.016$

1.77

1.36

2.30

$<0.001$

1.82

1.38

$2.40<0.001$

3.00

2.22

4.05

$<0.001$

Education (ref. $<9$ years)

$$
\begin{aligned}
& 9 \text { years } \\
& >9 \text { years }
\end{aligned}
$$

Urban residence (ref. rural)

0.87

0.67

$1.12 \quad 0.285$

$\begin{array}{lll}0.87 & 0.69 & 1.09\end{array}$

0.227

Smoking (ref. never)

$\begin{array}{llllllll}0.91 & 0.76 & 1.11 & 0.359 & 1.12 & 0.91 & 1.39 & 0.279 \\ 0.74 & 0.55 & 0.98 & 0.037 & 0.82 & 0.56 & 1.21 & 0.323 \\ 1.15 & 0.90 & 1.47 & 0.252 & 1.03 & 0.77 & 1.37 & 0.854\end{array}$




\begin{tabular}{|c|c|c|c|c|c|c|c|c|}
\hline Quit & 1.02 & 0.81 & 1.29 & 0.86 & 1.37 & 0.88 & 2.15 & 0.162 \\
\hline Smoke & 1.07 & 0.88 & 1.30 & 0.487 & 0.97 & 0.70 & 1.36 & 0.877 \\
\hline \multicolumn{9}{|l|}{ Alcohol consumption (ref. none) } \\
\hline Less than once a month & 1.02 & 0.78 & 1.34 & 0.889 & 1.02 & 0.74 & 1.41 & 0.882 \\
\hline Once a month or more frequently & 1.20 & 1.01 & 1.42 & 0.036 & 0.79 & 0.56 & 1.12 & 0.186 \\
\hline Self-rated health (ref. good) & 0.93 & 0.76 & 1.13 & 0.463 & 0.86 & 0.72 & 1.03 & 0.100 \\
\hline \multicolumn{9}{|l|}{ Depressive symptoms } \\
\hline Depressed & 0.91 & 0.76 & 1.09 & 0.327 & 0.96 & 0.82 & 1.14 & 0.656 \\
\hline Missing & 0.77 & 0.51 & 1.16 & 0.215 & 1.04 & 0.77 & 1.42 & 0.780 \\
\hline \multicolumn{9}{|l|}{ BMI (ref. < 18.5) } \\
\hline $18.5-23.99$ & 0.88 & 0.69 & 1.14 & 0.338 & 1.21 & 0.89 & 1.64 & 0.232 \\
\hline $24-27.99$ & 1.19 & 0.89 & 1.58 & 0.243 & 1.52 & 1.10 & 2.10 & 0.012 \\
\hline$\geq 28$ & 1.30 & 0.87 & 1.94 & 0.197 & 2.30 & 1.60 & 3.30 & $<0.001$ \\
\hline Missing & 1.58 & 1.07 & 2.35 & 0.023 & 1.11 & 0.54 & 2.25 & 0.782 \\
\hline
\end{tabular}

IRR: incidence rate ratio; CI: confidence interval 
${ }^{(1)}$ Social participation was defined by participants saying yes to participating in any of the following activities once a week or more frequently in the previous one month: interact with friends; play mahjong, chess or cards, or go to a community club; go to a sport, social, or other kind of club; take part in a community-related organization; or attend volunteer or charity work. 
Figure 1. CHARLS participant flow chart (2011-2013) $215 \times 282 \mathrm{~mm}(300 \times 300 \mathrm{DPI})$ 